Open Systems \& Information Dynamics

Vol. 22, No. 4 (2015) 1599001 (2 pages)

DOI:10.1142/S1230161215990012

\title{
Index of Vol. 22 (2015)
}

AbDel-Khalek S., Quantum Entanglement and Geometric Phase of Two Moving Two-Level Atoms

Accardi L. And A. Dhahri, Polynomial Extensions of the Weyl $C^{*}$-Algebra

Ay N., see Weis S., A. KnAuf, N. Ay, And M.-J. ZhaO

Benatti F. And L. GoubA, Semi-Classical Localisation Properties of Quantum Oscillators on a Noncommutative Configuration Space

BrÁDler K., The Pitfalls of Deciding Whether a Quantum Channel is (Conjugate) Degradable and How to Avoid Them

Choudhary A., see Kumar S. And A. Choudhary

Chruściński D., see Rutkowski A., G. SARBicki, And D. Chruściński

Daoud M., W. Kaydi, And H. El Hadfi, Quantum Discord in Photon-Added Glauber Coherent States of GHZ-Type

Dhahri A., see ACCARdi L. AND A. Dhahri

El Hadfi H., see DaOud M., W. Kaydi, And H. El Hadfi

Fagnola F. And R. Rebolledo, Entropy Production and Detailed Balance for a Class of Quantum Markov Semigroups

1550013

Falniowski F., Generalized Conditional Entropy — Determinicity of a Process and Rokhlin's Formula

1550025

Fidaleo F., A Note on Boolean Stochastic Processes

1550004

Frank T.D., see Kim S., J. M. Gordon, and T. D. Frank

1550007

Gordon J. M., see Kim S., J. M. Gordon, And T. D. Frank

1550007

Gouba L., see Benatti F. And L. GoubA

Gough J., The Global versus Local Hamiltonian Description of Quantum InputOutput Theory

Gregoratti M., The Hamiltonian Generating Quantum Stochastic Evolutions in the Limit from Repeated to Continuous Interactions

Havinga M., New Families of Complex Hadamard Matrices

1550017

IrIYAma S., M. OHYA, AND I. V. VolOvich, On Quantum Algorithm for Binary Search and Its Computational Complexity

Kamizawa T., On Functionally Commutative Quantum Systems

Kim S., J. M. Gordon, And T.D. Frank, Nonequilibrium Thermodynamic State Variables of Human Self-Paced Rhythmic Motions: Canonical-Dissipative Approach, Augmented Langevin Equation, and Entropy Maximization 
Author Index

Knauf A., see Weis S., A. Knauf, N. Ay, and M.-J. Zhao

Kumar S. And A. Choudhary, Generalized 'Useful' Relative Information Measures of Type $(\alpha, \beta)$

Liu F., see Sun J., S. F. Lu, And F. LiU

Lu S. F., see Sun J., S. F. Lu, AND F. LiU

Matolcsi M. AND M. Weiner, An Improvement on the Delsarte-Type LP-Bound with Application to MUBs

1550001

Miller M. And R. Olkiewicz, Stable Subspaces of Positive Maps of Matrix Algebras

1550011

Mukhamedov F., On Pure Quasi-Quantum Quadratic Operators of $\mathbb{M}_{2}(\mathbb{C})$ II

Nicolis G. AND S. C. Nicolis, Probabilistic Network Approach to DecisionMaking

1550012

Nicolis S. C., see Nicolis G. AND S. C. Nicolis

1550012

Ohya M., see Iriyama S., M. Ohya, and I. V. Volovich

1550019

Olkiewicz R., see Miller M. and R. Olkiewicz

1550011

Ostrowski M., Quantum Simulation of the Dirac Particle

1550002

Rastegin A.E., On Uncertainty Relations and Entanglement Detection with Mutually Unbiased Measurements

1550005

Rebolledo R., see Fagnola F. and R. Rebolledo

1550013

Rutkowski A., G. SARBicki, and D. Chruściński, A Class of Bistochastic Positive Optimal Maps in $M_{d}(\mathbb{C})$

1550016

SArbicki G., see Rutkowski A., G. SArbicki, and D. Chruściński

1550016

Sun J., S. F. Lu, AND F. Liu, A Modified Adiabatic Quantum Algorithm for Evaluation of Boolean Functions

1550018

Volovich I. V., see Iriyama S., M. Ohya, and I. V. Volovich

1550019

Watanabe N., A Note on Entropies for Compound Systems

1550010

Weiner M., see Matolcsi M. AND M. Weiner

1550001

Weis S., A. Knauf, N. Ay, And M.-J. ZhaO, Maximizing the Divergence from a Hierarchical Model of Quantum States

1550006

Werpachowska A., Reduced Operator Approximation for Modelling Open Quantum Systems

1550008

Zhao M.-J., see Weis S., A. Knauf, N. Ay, and M.-J. Zhao

1550006 